TRANSACTIONS OF THE

AMERICAN MATHEMATICAL SOCIETY

Volume 361, Number 3, March 2009, Pages 1645-1665

S 0002-9947(08)04625-4

Article electronically published on October 22, 2008

\title{
A WEIERSTRASS-TYPE THEOREM FOR HOMOGENEOUS POLYNOMIALS
}

\author{
DAVID BENKO AND ANDRÁS KROÓ
}

\begin{abstract}
By the celebrated Weierstrass Theorem the set of algebraic polynomials is dense in the space of continuous functions on a compact set in $\mathbb{R}^{d}$. In this paper we study the following question: does the density hold if we approximate only by homogeneous polynomials? Since the set of homogeneous polynomials is nonlinear, this leads to a nontrivial problem. It is easy to see that: 1) density may hold only on star-like $\mathbf{0}$-symmetric surfaces; 2) at least 2 homogeneous polynomials are needed for approximation. The most interesting special case of a star-like surface is a convex surface. It has been conjectured by the second author that functions continuous on $\mathbf{0}$-symmetric convex surfaces in $\mathbb{R}^{d}$ can be approximated by sums of 2 homogeneous polynomials. This conjecture has not yet been resolved, but we make substantial progress towards its positive settlement. In particular, it is shown in the present paper that the above conjecture holds for 1) $d=2$;2) convex surfaces in $\mathbb{R}^{d}$ with $C^{1+\epsilon}$ boundary.
\end{abstract}

\section{INTRODUCTION}

The celebrated theorem of Weierstrass on the density of real algebraic polynomials in the space of real continuous functions on an interval $[a, b]$ is one of the main results in analysis. Its generalization for real multivariate polynomials was given by Picard; subsequently the Stone-Weierstrass theorem led to the extension of these results for subalgebras in $C(K)$.

In this paper we shall consider the question of density of homogeneous polynomials. Homogeneous polynomials are a standard tool appearing in many areas of analysis, so the question of their density in the space of continuous functions is a natural problem. Clearly, the set of homogeneous polynomials is substantially smaller relative to all algebraic polynomials. More importantly, this set is nonlinear, so its density cannot be handled via the Stone-Weierstrass theorem. Furthermore, due to the special structure of homogeneous polynomials some restrictions should be made on the sets where we want to approximate (they have to be star-like), and at least 2 polynomials are always needed for approximation (an even and an odd one).

Received by the editors October 14, 2005 and, in revised form, April 24, 2007.

2000 Mathematics Subject Classification. Primary 41A10, 31A05; Secondary 52A10, 52A20.

Key words and phrases. Weierstrass, uniform approximation, homogeneous polynomials, convex body.

The second author was supported by the OTKA grant \# T049196. This research was partially written during this author's stay at the Center for Constructive Approximation, Vanderbilt University, Nashville, Tennessee.

(C)2008 American Mathematical Society Reverts to public domain 28 years from publication 
During the 5-th International Conference on Functional Analysis and Approximation Theory (Maratea, Italy, 2004) the second author proposed the following conjecture.

Conjecture 1. Let $K \subset \mathbb{R}^{d}$ be a convex body which is centrally symmetric to the origin. Then for any function $f$ continuous on the boundary $B d(K)$ of $K$ and any $\epsilon>0$ there exist two homogeneous polynomials $h$ and $g$ such that $|f-h-g| \leq \epsilon$ on $B d(K)$.

From now on we agree on the terminology that by "centrally symmetric" we mean "centrally symmetric to the origin".

Subsequently in 4 the authors verified the above conjecture for crosspolytopes in $\mathbb{R}^{d}$ and arbitrary convex polygons in $\mathbb{R}^{2}$.

In this paper we shall verify the conjecture for those convex bodies in $\mathbb{R}^{d}$ whose boundary $B d(K)$ is $C^{1+\epsilon}$ for some $0<\epsilon \leq 1$ (Theorem 2). Moreover, the conjecture will be verified in its full generality for $d=2$ (Theorem 3).

It should be noted that parallel to our investigations P. Varjú 13 also proved the conjecture for $d=2$. In addition, he gives in [13 an affirmative answer to the conjecture for arbitrary centrally symmetric polytopes in $\mathbb{R}^{d}$, and for those convex bodies in $\mathbb{R}^{d}$ whose boundary is $C^{2}$ and has positive curvature. We would also like to point out that our method of verifying the conjecture for $d=2$ is based on potential theory and is different from the approach taken in [13] (which is also based on potential theory). Likewise our method of treating $C^{1+\epsilon}$ convex bodies is different from the approach used in [13] for $C^{2}$ convex bodies with positive curvature.

\section{MAIN RESULTS}

Let $K$ be a centrally symmetric convex body in $\mathbb{R}^{d}$. We may assume that $2 \leq d$ and $\operatorname{dim}(K)=d$. The boundary of $K$ is $B d(K)$, which is given by the representation

$$
B d(K):=\left\{\mathbf{u} r(\mathbf{u}): \mathbf{u} \in S^{d-1}\right\}
$$

where $r$ is a positive even real-valued function on $S^{d-1}$. Here $S^{d-1}$ stands for the unit sphere in $\mathbb{R}^{d}$. We shall say that $K$ is $C^{1+\epsilon}$, written $K \in C^{1+\epsilon}$, if the first partial derivatives of $r$ are Hölder continuous with Hölder index $\epsilon>0$ on the unit sphere. Furthermore denote by

$$
H_{n}^{d}:=\left\{\sum_{k_{1}+\ldots+k_{d}=n} c_{\mathbf{k}} \mathbf{x}^{\mathbf{k}}: c_{\mathbf{k}} \in \mathbb{R}\right\}
$$

the space of real homogeneous polynomials of degree $n$ in $\mathbb{R}^{d}$. Our first main result is the following.

Theorem 2. Let $K \in C^{1+\epsilon}$ be a centrally symmetric convex body in $\mathbb{R}^{d}$, where $0<\epsilon \leq 1$. Then for every $f \in C(B d(K))$ there exist $h_{n} \in H_{n}^{d}+H_{n-1}^{d}, n \in \mathbb{N}$, such that $h_{n} \rightarrow f$ uniformly on $B d(K)$ as $n \rightarrow \infty$.

Thus Theorem 2 gives an affirmative answer to the conjecture under the additional condition of $C^{1+\epsilon}$ smoothness of the convex surface. For $d=2$ we can verify the conjecture in its full generality. Thus we shall prove the following.

Theorem 3. Let $K$ be a centrally symmetric convex body in $\mathbb{R}^{2}$. Then for every $f \in C(B d(K))$ there exist $h_{n} \in H_{n}^{2}+H_{n-1}^{2}, n \in \mathbb{N}$, such that $h_{n} \rightarrow f$ uniformly on $B d(K)$ as $n \rightarrow \infty$. 
We shall see that Theorem 3 follows from

Theorem 4. Let $1 / W(x)$ be a positive convex function on $\mathbb{R}$ such that $|x| / W(1 / x)$ is also positive and convex. Let $g(x)$ be a continuous function which has the same limits at $-\infty$ and at $+\infty$. Then we can approximate $g(x)$ uniformly on $\mathbb{R}$ by weighted polynomials $W(x)^{n} p_{n}(x), n=0,2,4, \ldots, \operatorname{deg} p_{n} \leq n$.

\section{Proof of Theorem 2}

The proof of Theorem 2 will be based on several lemmas. The main auxiliary result is the next lemma which provides an estimate for the approximation of unity by even homogeneous polynomials. In what follows $\|\ldots\|_{D}$ stands for the uniform norm on $D$.

Our main lemma to prove Theorem 2 is the following.

Lemma 5. Let $\tau \in(0,1)$. Under conditions of Theorem 2 there exist $h_{2 n} \in$ $H_{2 n}^{d}, n \in \mathbb{N}$, such that

$$
\left\|1-h_{2 n}\right\|_{B d(K)}=o\left(n^{-\tau \epsilon}\right) .
$$

We shall first state and verify three technical lemmas (Lemmas 6] 8); then the proof of Lemma 5 will be given. Finally it will be shown that Theorem 2 follows easily from Lemma 5 .

The following lemma provides a partition of unity which we shall need below. In what follows a cube in $\mathbb{R}^{d}$ is called regular if all its edges are parallel to the coordinate axes. We denote the set $\{0,1,2, \ldots\}^{d}$ by $\mathbb{Z}_{+}^{d}$.

Lemma 6. Given $0<h \leq 1$ there exist nonnegative even functions $g_{\mathbf{k}} \in C^{\infty}\left(\mathbb{R}^{d}\right)$ such that their support consists of at most $2^{d}$ regular cubes with edge $h$. Moreover at most $2^{d}$ of supports of $g_{\mathbf{k}}$ have nonempty intersection, and

$$
\begin{gathered}
\sum_{\mathbf{k} \in \mathbb{Z}_{+}^{d}} g_{\mathbf{k}}(\mathbf{x})=1, \quad \mathbf{x} \in \mathbb{R}^{d}, \\
\left|\partial^{m} g_{\mathbf{k}}(\mathbf{x}) / \partial x_{j}^{m}\right| \leq c / h^{m}, \quad \mathbf{x} \in \mathbb{R}^{d}, m \in \mathbb{Z}_{+}^{1}, 1 \leq j \leq d,
\end{gathered}
$$

where $c>0$ depends only on $m \in \mathbb{Z}_{+}^{1}$ and $d$.

Proof. The main step of the proof consists of verifying the lemma for $d=1$. Let $g \in C^{\infty}(\mathbb{R})$ be an odd function on $\mathbb{R}$ such that $g=1$ for $x<-1 / 2$ and monotone decreasing from 1 to 0 on $(-1 / 2,0)$. Further, let $g^{*}(x)$ be an even function on $\mathbb{R}$ such that $g^{*}(x)$ equals $g(x-3 / 2) / 4+3 / 4$ on [0,2], and $g(x-5 / 2) / 4+1 / 4$ for $x>2$. Then it is easy to see that $g^{*} \in C^{\infty}(\mathbb{R})$, it equals 1 on $[-1,1], 0$ for $|x|>3$ and is monotone decreasing on $[1,3]$. Moreover

$$
g^{*}(x)+g^{*}(x-4)=1, \quad x \in[-1,5] .
$$

Now set

$$
g_{0}(x):=\frac{1}{2} g^{*}(x), \quad g_{k}(x):=g^{*}(x-4 k)+g^{*}(x+4 k), \quad k \in \mathbb{Z}_{+}^{1}, \quad k \neq 0 .
$$

Then the $g_{k}$ 's are even functions which by (4) satisfy the relation

$$
\sum_{k=0}^{\infty} g_{k}(x)=1, \quad x \in \mathbb{R} .
$$


In addition, the support of $g_{k}$ equals $\pm[-3+4 k, 3+4 k]$ and at most 2 of the $g_{k}$ 's can be nonzero at any given $x \in \mathbb{R}$. Finally, for a fixed $0<h \leq 1, \mathbf{x} \in \mathbb{R}^{d}$ and $\mathbf{k}=\left(k_{1}, \ldots, k_{d}\right) \in \mathbb{Z}_{+}^{d}$ set

$$
g_{\mathbf{k}}(\mathbf{x}):=\prod_{j=1}^{d} g_{k_{j}}\left(6 x_{j} / h\right) .
$$

It is easy to see that these functions give the needed partition of unity.

For the centrally symmetric convex body $K$ let

$$
|\mathbf{x}|_{K}:=\inf \{a>0: \mathbf{x} / a \in K\}
$$

be its Minkowski functional and set

$$
\delta_{K}:=\sup \left\{|\mathbf{x}| /|\mathbf{x}|_{K}: \mathbf{x} \in \mathbb{R}^{d}\right\}=\max \{|\mathbf{x}|: \mathbf{x} \in B d(K)\} .
$$

Moreover for $\mathbf{a} \in B d(K)$ denote by $L_{\mathbf{a}}$ a supporting hyperplane at $\mathbf{a}$.

Lemma 7. Let $\mathbf{a} \in B d(K), h_{n} \in H_{2 n}^{d}$ be such that for any $\mathbf{x} \in L_{\mathbf{a}},|\mathbf{x}-\mathbf{a}| \leq 4 \delta_{K}$ we have $\left|h_{n}(\mathbf{x})\right| \leq 1$. Then whenever $\mathbf{x} \in L_{\mathbf{a}}$ satisfies $|\mathbf{x}-\mathbf{a}|>4 \delta_{K}$ and $\mathbf{x} / t \in K$ we have

$$
\left|h_{n}(\mathbf{x} / t)\right| \leq(2 / 3)^{2 n} .
$$

Proof. Clearly the conditions of the lemma yield that whenever $|\mathbf{x}-\mathbf{a}|>4 \delta_{K}$

(5) $1 /|\mathbf{x}|_{K} \leq \delta_{K} /|\mathbf{x}| \leq \delta_{K} /(|\mathbf{x}-\mathbf{a}|-|\mathbf{a}|) \leq \delta_{K} /\left(|\mathbf{x}-\mathbf{a}|-\delta_{K}\right) \leq 4 \delta_{K} / 3|\mathbf{x}-\mathbf{a}|$.

It is well known that for any univariate polynomial $p$ of degree at most $n$ such that $|p| \leq 1$ in $[-a, a]$, it holds that $|p(x)| \leq(2 x / a)^{n}$ whenever $|x|>a$. Therefore using

(5) and the assumption imposed on $h_{n}$ we have

$$
\left|h_{n}(\mathbf{x})\right| \leq\left(2|\mathbf{x}-\mathbf{a}| / 4 \delta_{K}\right)^{2 n} \leq\left(2|\mathbf{x}|_{K} / 3\right)^{2 n} .
$$

Now it remains to note that by $\mathbf{x} / t \in K$ it follows that $|\mathbf{x}|_{K} \leq|t|$, and thus we obtain (3) from (6). This completes the proof of the lemma.

Lemma 8. Consider the functions $g_{\mathbf{k}}$ from Lemma 6 . Then for at most $8^{d} / 2 h^{d}$ of them their support has nonempty intersection with $S^{d-1}$.

Proof. Recall that the support of the $g_{\mathbf{k}}$ 's consists of regular cubes with edge $h \leq 1$, so if $A_{\mathbf{k}}:=\operatorname{supp}\left(g_{\mathbf{k}}\right)$ has nonempty intersection with the unit sphere $S^{d-1}$, then $A_{\mathbf{k}} \subset D$, where $D$ stands for the regular cube centered at 0 with edge 4 . Now let $f_{\mathbf{k}}$ be the characteristic function of $A_{\mathbf{k}}$. Since at most $2^{d}$ of the $A_{\mathbf{k}}$ 's have a nonempty intersection it follows that

$$
\sum f_{\mathbf{k}}(\mathbf{x}) \leq 2^{d}, \mathbf{x} \in \mathbb{R}^{d} .
$$

Moreover, $m\left(A_{\mathbf{k}}\right)=2 h^{d}$, where $m($.$) stands for the Lebesgue measure in \mathbb{R}^{d}$. Using (7) we have that

$$
\sum \int_{D} f_{\mathbf{k}} d m \leq 2^{d} m(D)=8^{d}
$$

Since

$$
\int_{D} f_{\mathbf{k}} d m=m\left(A_{\mathbf{k}}\right)=2 h^{d}
$$

whenever $A_{\mathbf{k}} \subset D$, the statement of the lemma easily follows from (8). 
Proof of Lemma 5. Denote by $g_{k}, 1 \leq k \leq N$ those functions from Lemma 6 whose support $A_{k}$ has a nonempty intersection with $S^{d-1}$. Then by Lemma 8

$$
N \leq(c / h)^{d} .
$$

Moreover, by (1)

$$
\sum_{k=1}^{N} g_{k}=1 \text { on } S^{d-1} .
$$

By construction of Lemma 6 the support of each $g_{k}$ (except $g_{0}$ ) consists of at most $2^{d-1}$ symmetric pairs of cubes with edge $h$. Thus without loss of generality we may assume that $A_{k}$ consists of 2 symmetric disjoint cubes. (We also need to assume that $h<\sqrt{d-1}$ so that $\operatorname{supp}\left(g_{0}\right) \cap S^{d-1}=\emptyset$.)

Set

$$
B_{k}:=A_{k} \cap S^{d-1}, \quad C_{k}:=\left\{\mathbf{u} r(\mathbf{u}): \mathbf{u} \in B_{k}\right\} \subset B d(K), \quad 1 \leq k \leq N .
$$

For each $1 \leq k \leq N$ choose a point $\mathbf{u}_{k} \in B_{k}$ and set $\mathbf{x}_{k}:=\mathbf{u}_{k} r\left(\mathbf{u}_{k}\right) \in B d(K)$. Furthermore let $L_{k}$ be the supporting plane to $B d(K)$ at the point $\mathbf{x}_{k}$ and set for $1 \leq k \leq N, L_{k}^{*}:=L_{k} \cup\left(-L_{k}\right)$

$$
\begin{gathered}
D_{k}:=\left\{\mathbf{x} \in L_{k}^{*}: \mathbf{x}=t \mathbf{u} \text { for some } \mathbf{u} \in B_{k}, t>0\right\} \\
f_{k}(\mathbf{x}):=g_{k}(\mathbf{u}), \quad \mathbf{x} \in B d(K), \mathbf{x}=\mathbf{u} r(\mathbf{u}), \quad \mathbf{u} \in S^{d-1}, \\
q_{k}(\mathbf{x}):=g_{k}(\mathbf{u}), \quad \mathbf{x} \in L_{k}^{*}, \mathbf{x}=t \mathbf{u}, \quad \mathbf{u} \in S^{d-1}, \quad t>0 .
\end{gathered}
$$

Clearly, $q_{k} \in C^{\infty}\left(L_{k}^{*}\right)$ is an even positive function which by property (2) can be extended to a regular centrally symmetric cube $I \supset K$ so that we have on $I$

$$
\left|\partial^{m} q_{k} / \partial x_{j}^{m}\right| \leq c / h^{m}, \quad 1 \leq j \leq d, \quad 1 \leq k \leq N .
$$

Here and in what follows we denote by $c$ (possibly distinct) positive constants depending only on $d, m$ and $K$. We can assume that $I$ is sufficiently large so that

$$
I \supset G_{k}:=\left\{\mathbf{x} \in L_{k}:\left|\mathbf{x}-\mathbf{x}_{k}\right| \leq 4 \delta_{K}\right\}, \quad 1 \leq k \leq N .
$$

Then by the multivariate Jackson Theorem (see e.g. [10 ) applied to the even functions $q_{k}$ satisfying (11) for arbitrary $m \in \mathbb{N}$ (to be specified below), there exist even multivariate polynomials $p_{k}$ of total degree at most $2 n$ such that

$$
\left\|q_{k}-p_{k}\right\|_{G_{k}^{*}} \leq c /(h n)^{m} \leq 1, \quad 1 \leq k \leq N,
$$

where $G_{k}^{*}:=G_{k} \cup\left(-G_{k}\right)$, and $n$ is sufficiently large.

We now claim that without loss of generality it may be assumed that each $p_{k}$ is in $H_{2 n}^{d}$. Indeed, since $G_{k}^{*} \subset L_{k}^{*}$ it follows that the homogeneous polynomial $h_{2}:=\langle\mathbf{x}, \mathbf{w}\rangle^{2} \in H_{2}^{d}$ is identically equal to 1 on $G_{k}^{*}$ (here $\mathbf{w}$ is a properly normalized normal vector to $L_{k}$ ), so multiplying the even degree monomials of $p_{k}$ by even powers of $h_{2}$ we can replace $p_{k}$ by a homogeneous polynomial from $H_{2 n}^{d}$ so that (12) holds. Thus we may assume that $p_{k} \in H_{2 n}^{d}$ and relations (12) hold. In particular, (12) also yields that

$$
\left\|p_{k}\right\|_{G_{k}^{*}} \leq 2, \quad 1 \leq k \leq N .
$$


Now consider an arbitrary $\mathbf{x} \in B d(K) \backslash C_{k}$. Then with some $t>1$ we have $t \mathbf{x} \in L_{k}^{*}$ and $q_{k}(t \mathbf{x})=0$. Hence if $t \mathbf{x} \in G_{k}^{*}$, then by (12) it follows that

$$
\left|p_{k}(\mathbf{x})\right| \leq\left|p_{k}(t \mathbf{x})\right| \leq c /(h n)^{m} .
$$

On the other hand if $t \mathbf{x} \notin G_{k}^{*}$, then by (13) and Lemma 7 we obtain

$$
\left|p_{k}(\mathbf{x})\right| \leq 2(2 / 3)^{2 n} \text {. }
$$

The last two estimates yield that for every $\mathrm{x} \in B d(K) \backslash C_{k}$ we have

$$
\left|p_{k}(\mathbf{x})\right| \leq c\left((2 / 3)^{2 n}+(h n)^{-m}\right), \quad 1 \leq k \leq N .
$$

Now let us assume that $\mathbf{x} \in C_{k}$.

Clearly, the $C^{1+\epsilon}$ property of $B d(K)$ yields that whenever $\mathbf{x} \in B d(K), t \mathbf{x} \in$ $L_{k}^{*}, t>1$, we have for every $1 \leq k \leq N$

$$
(t-1)|\mathbf{x}|=|\mathbf{x}-t \mathbf{x}| \leq c \min \left\{\left|\mathbf{x}-\mathbf{x}_{k}\right|,\left|\mathbf{x}+\mathbf{x}_{k}\right|\right\}^{1+\epsilon} .
$$

Obviously, for every $\mathbf{u} \in B_{k}$

$$
\min \left\{\left|\mathbf{u}-\mathbf{u}_{k}\right|,\left|\mathbf{u}+\mathbf{u}_{k}\right|\right\} \leq \sqrt{d} h .
$$

This and (15) yields that for $\mathbf{u} \in B_{k}, \mathbf{x}=\mathbf{u} r(\mathbf{u}) \in C_{k}, t \mathbf{x} \in D_{k}(c>t>1)$ we have for $1<t<c, 0<h<c$

$$
t-1 \leq c h^{1+\epsilon}, \quad D_{k} \subset G_{k}^{*}, 0<h<h_{0} .
$$

Hence using (12), (13) and (16) we obtain for $0<h^{1+\epsilon} \leq c n^{-1}, 1 \leq k \leq N$,

$$
\begin{gathered}
\left|f_{k}(\mathbf{x})-p_{k}(\mathbf{x})\right|=\left|q_{k}(t \mathbf{x})-p_{k}(\mathbf{x})\right| \leq\left|q_{k}-p_{k}\right|(t \mathbf{x})+\left|p_{k}(t \mathbf{x})-p_{k}(\mathbf{x})\right| \\
\leq c /(h n)^{m}+\left|p_{k}(\mathbf{x})\right|\left(t^{2 n}-1\right) \leq c\left((h n)^{-m}+n h^{1+\epsilon}\right), \quad \mathbf{x} \in C_{k} .
\end{gathered}
$$

Denote for $\mathbf{x} \in B d(K)$

$$
R(\mathbf{x}):=\left\{k: \mathbf{x} \in C_{k}\right\}, \quad \# R(\mathbf{x}) \leq 2^{d} .
$$

Then using the above relation together with (10), (17), (14) and (9) we obtain for every $\mathbf{x} \in B d(K)$

$$
\begin{gathered}
\left|1-\sum_{k=1}^{N} p_{k}(\mathbf{x})\right|=\left|\sum_{k=1}^{N}\left(f_{k}-p_{k}\right)(\mathbf{x})\right| \leq\left|\sum_{k \in R(\mathbf{x})} \ldots\right|+\left|\sum_{k \notin R(\mathbf{x})} \ldots\right| \\
\leq c 2^{d}\left(1 /(h n)^{m}+n h^{1+\epsilon}\right)+N|| p_{k} \|_{B d(K) \backslash C_{k}} \\
\leq c\left(h^{-m-d} n^{-m}+h^{-d}(2 / 3)^{2 n}+n h^{1+\epsilon}\right) .
\end{gathered}
$$

Now it remains to choose proper values for $m$ and $h$.

Choose $m \in \mathbb{N}$ to be so large that

$$
R:=\frac{m \epsilon-d}{1+m+\epsilon+d}>\tau \epsilon \text { and let } \gamma:=\frac{1+m}{1+m+\epsilon+d} .
$$

Letting $h:=n^{-\gamma}$ we see that $h^{-m-d} n^{-m}=n h^{1+\epsilon}=n^{-R}$. (Hence the $h^{1+\epsilon} \leq c n^{-1}$ condition is satisfied.) In addition $h^{-d}(2 / 3)^{2 n}=O\left(n^{-R}\right)$, too. This completes the proof of Lemma 5

Proof of Theorem 2. First we use the classical Weierstrass Theorem to approximate $f \in C(B d(K))$ by a polynomial

$$
p_{m}=\sum_{j=0}^{m} h_{j}^{*}, \quad h_{j}^{*} \in H_{j}^{d}, \quad 0 \leq j \leq m,
$$


of degree at most $m$ so that

$$
\left\|f-p_{m}\right\|_{B d(K)} \leq \delta
$$

with any given $\delta>0$. Let $\tau \in(0,1)$ be arbitrary. According to Lemma 5 there exist $h_{n, j} \in H_{2 n-2[j / 2]}^{d}$ such that $\left\|1-h_{n, j}\right\|_{B d(K)}=O\left(n^{-\tau \epsilon}\right), 0 \leq j \leq m$. Clearly,

$$
h^{*}:=\sum_{j=0}^{m} h_{n, j} h_{j}^{*} \in H_{2 n}^{d}+H_{2 n+1}^{d}
$$

and

$$
\left\|f-h^{*}\right\|_{B d(K)} \leq \delta+O\left(n^{-\tau \epsilon}\right) .
$$

\section{Proof of Theorems 3 and 4}

First we are going to prove Theorem 4, Then at the end of this section we will show the strong connection between approximation by homogeneous polynomials and approximation by weighted polynomials, and prove Theorem 3 .

Let $\overline{\mathbb{R}}:=\mathbb{R} \cup\{\infty\}$ denote the one-point compactified real line (whose topology is isomorphic to the topology of the unit circle). (Remark: $\infty$ and $+\infty$ are not the same.)

Let $w(x) \not \equiv 0$ be a nonnegative continuous function on $\overline{\mathbb{R}}$ such that

$$
\lim _{x \rightarrow \infty}|x| w(x)=\alpha \in[0,+\infty) \text { exists. }
$$

Approximation by weighted polynomials of the form $w(x)^{n} p_{n}(x)$ was introduced by Saff, [7]. It was proved by Kuijlaars ([5], see also [8], Theorem VI.1.1) that when $\alpha=0$, there exists a closed set $Z(w) \subset \mathbb{R}$ with the property that a continuous function $f(x), x \in \mathbb{R}$, is the uniform limit of weighted polynomials $w^{n} P_{n}$ $(n=0,1,2, \ldots)$ on $\mathbb{R}$ if and only if $f(x)$ vanishes on $Z(w)$. (One can also see that $f(x)$ must have compact support. But this is no longer true in the next lemma.) We formulate the following version of the aforementioned theorem.

Lemma 9. Assume that $0 \leq \alpha$ at (19). Then there exists a closed set $Z_{\overline{\mathbb{R}}}(w)$ such that a continuous function $f(x), x \in \overline{\mathbb{R}}$, is the uniform limit of weighted polynomials $w^{n} p_{n}(n=0,2,4, \ldots)$ on $\overline{\mathbb{R}}$ if and only if $f(x)$ vanishes on $Z_{\overline{\mathbb{R}}}(w)$.

Proof. Let $X:=\overline{\mathbb{R}}$. Note that $w^{n} p_{n}$ is continuous on $\overline{\mathbb{R}}$ when $n$ is even. (Naturally the value $\left(w^{n} p_{n}\right)(\infty)$ is defined to be $\lim _{x \rightarrow \pm \infty}\left(w^{n} p_{n}\right)(x)$.)

Let $\mathcal{A}$ be the collection of continuous functions $f$ on $X$ such that $w^{n} p_{n} \rightarrow f$ $(n=0,2,4, \ldots)$ uniformly on $X$ for some $p_{n}$. Define the set $Z_{\overline{\mathbb{R}}}(w):=\{x \in X:$ $f(x)=0$ for all $f \in \mathcal{A}\}$, which is certainly closed.

It is easy to see (similarly as in [8], Theorem VI.1.1) that $\mathcal{A}$ is an algebra which is closed under uniform limits. Also, it separates points in the sense that if $x_{1}, x_{2} \in$ $X \backslash Z_{\overline{\mathbb{R}}}(w)$ are two distinct points, then there exists $f \in \mathcal{A}$ such that $f\left(x_{1}\right) \neq f\left(x_{2}\right)$. Indeed, let us assume that, say, $x_{2}$ is finite and let $g \in \mathcal{A}$ such that $g\left(x_{1}\right) \neq$ 0 . Let $w^{n} p_{n} \rightarrow g(n=0,2,4, \ldots)$ uniformly on $X$. Clearly, $w\left(x_{1}\right) \neq 0$. Also $w^{n+2}(x)\left[\left(x-x_{2}\right)^{2} p_{n}(x)\right] \rightarrow w^{2}(x)\left(x-x_{2}\right)^{2} g(x)=: f(x)(n=0,2,4, \ldots)$ uniformly on $X$ because $\left\|w^{2}(x)\left(x-x_{2}\right)^{2}\right\|_{\overline{\mathbb{R}}}<+\infty$. Thus $f(x) \in \mathcal{A}$. Also, $f\left(x_{1}\right) \neq 0=f\left(x_{2}\right)$ (which holds even if $x_{1}$ was infinity).

Since $\mathcal{A}$ satisfies the properties above, by the Stone-Weierstrass Theorem

$$
\mathcal{A}=\left\{f: f \text { is continuous on } X \text { and } f \equiv 0 \text { on } Z_{\overline{\mathbb{R}}}(w)\right\} .
$$


The following definitions and facts are well known in logarithmic potential theory (see [8] and [9]).

Let $w(x) \not \equiv 0$ be a nonnegative continuous function on $\overline{\mathbb{R}}$ satisfying (19). When $\alpha=0$, then $w$ belongs to the class of so called "admissible" weights.

We write $w(x)=\exp (-q(x))$ and call $q(x)$ an external field. If $\mu$ is a positive Borel unit measure on $\overline{\mathbb{R}}$, in short a "probability measure", then its weighted energy is defined by

$$
I_{w}(\mu):=\iint \log \frac{1}{|x-y| w(x) w(y)} d \mu(x) d \mu(y) .
$$

The integrand is bounded from below $\left(9\right.$, p. 3), so $I_{w}(\mu)$ is well defined and $-\infty<I_{w}(\mu)$. Whenever it makes sense, we define the (unweighted) logarithmic energy of $\mu$ as $I_{1}(\mu)$, where 1 denotes the constant 1 function. There exists a unique probability measure $\mu_{w}$ - called the equilibrium measure associated with $w$ - which minimizes $I_{w}(\mu)$. Also,

$$
V_{w}:=I_{w}\left(\mu_{w}\right) \quad \text { is finite, }
$$

and $\mu_{w}$ has finite logarithmic energy when $\alpha=0$.

If the support of $\mu$ is compact, we define its potential as

$$
U^{\mu}(x):=\int \log \frac{1}{|t-x|} d \mu(t) .
$$

This definition makes sense for a signed measure $\nu$, too, if $\int|\log | t-x|| d|\nu|(t)$ exists.

Let

$$
S_{w}:=\operatorname{supp}\left(\mu_{w}\right) \text { denote the support of } \mu_{w} .
$$

When $\alpha=0$, then $S_{w}$ is a compact subset of $\mathbb{R}$. In this case with some $F_{w}$ constant we have

$$
U^{\mu_{w}}(x)+Q(x)=F_{w}, \quad x \in S_{w} .
$$

Functions with smooth integrals was introduced by Totik in [11.

Definitions 10. Let $f: R \rightarrow \mathbb{R} \cup\{-\infty\} \cup\{+\infty\}$ be a measurable function defined a.e. on a compact interval $R$. We say that $f$ has a smooth integral on $R$ if $f$ is nonnegative a.e. on $R$ and

$$
\int_{I} f=(1+o(1)) \int_{J} f
$$

where $I, J \subset R$ are any two adjacent intervals, both of which has length $0<\epsilon$, and $\epsilon \rightarrow 0$. The $o(1)$ term depends on $\epsilon$ and not on $I$ and $J$.

We say that a family of functions $\mathcal{F}$ has a uniformly smooth integral on $R$, if any $f \in \mathcal{F}$ is nonnegative a.e. on $R$ and (20) holds, where the $o(1)$ term depends on $\epsilon$ only, and not on the choice of $f, I$ or $J$.

Clearly, if $f$ is continuous and it has a positive lower bound on $R$, then $f$ has a smooth integral on $R$. Also, nonnegative linear combination of finitely many functions with smooth integrals on $R$ has also smooth integral on $R$.

Let $\mathcal{B}$ denote the Borel sets of $[-1,1]$ and let $\mathcal{L}$ denote the Lebesgues measurable sets of $R$. From the Fubini Theorem it follows that if $\nu$ is a finite positive Borel 
measure on $[-1,1]$ and $\left\{v_{t}(x): t \in[-1,1]\right\}$ is a family of functions with a uniformly smooth integral on $R$ for which $(t, x) \mapsto v_{t}(x)$ is a $(\mathcal{B}, \mathcal{L})$-measurable function, then

$$
v(x):=\int_{[-1,1]} v_{t}(x) d \nu(t)
$$

also has a smooth integral on $R$.

Finally, if $f_{n} \rightarrow f$ uniformly a.e. on $R, f_{n}$ has a smooth integral on $R$ and $f$ has positive lower bound a.e. on $R$, then $f$ has a smooth integral on $R$.

The notion of a smooth integral is very useful due to the following theorem of Totik, [11. We will use this theorem later in our proof. The theorem is formulated for admissible weights (so $\alpha=0$ ).

Theorem 11. Let $w$ be an admissible continuous weight. Let us suppose that $[a, b]$ is a subset of the support $S_{w}$, and the equilibrium measure has a density $v$ on $[a, b]$ that has a positive lower bound and smooth integral there. Then $(a, b) \cap Z(w)=\emptyset$.

Now we give some more definitions.

Definitions 12. Let $L \subset \mathbb{R}$ and let $f: L \rightarrow \mathbb{R} \cup\{-\infty\} \cup\{+\infty\}$ be a function which is defined almost everywhere (a.e.) on $L$. We say that $f$ is increasing if $f(x) \leq f(y)$ whenever $f$ is defined at $x$ and $y$ and $x \leq y$. We say that $f$ is increasing almost everywhere if there exists $L^{*} \subset L$ such that $L \backslash L^{*}$ has Lebesgue measure zero, $f(x)$ is defined for all $x \in L^{*}$ and $f(x) \leq f(y)$ whenever $x, y \in L^{*}, x \leq y$.

We say that $f$ is convex if $f$ is absolutely continuous, and $f^{\prime}(x)$ (which exists a.e.) is increasing a.e. on $L$.

We say that a property is satisfied inside $\mathbb{R}$ if it is satisfied on all compact subsets of $\mathbb{R}$.

Let $W: \overline{\mathbb{R}} \rightarrow \mathbb{R}$ be a nonnegative function. Define $Q: \overline{\mathbb{R}} \rightarrow(-\infty,+\infty]$ by

$$
W(t)=\exp (-Q(t))
$$

In the rest of the paper we will have the following assumptions on the weight $W(t), t \in \overline{\mathbb{R}}:$

$$
\begin{aligned}
& \frac{1}{W(t)} \text { is positive and convex on } \mathbb{R} \text {, } \\
& \frac{|t|}{W\left(-\frac{1}{t}\right)} \text { is positive and convex on } \mathbb{R} \text {. }
\end{aligned}
$$

(Naturally (22) is satisfied iff $|t| / W(1 / t)$ is positive and convex, which was the assumption in Theorem (4). When $t=0$ at (22), we define $-1 / 0$ to be $\infty$. We provide the function $W(t)=\left(1+|t|^{m}\right)^{-1 / m}, 1 \leq m$, as an example which satisfies (21) and (22).

Remark 13. Equivalently, instead of (22) we may assume that (23) below holds and $\lim _{t \rightarrow+\infty} t\left(t Q^{\prime}(t)-1\right) \leq \lim _{t \rightarrow-\infty} t\left(t Q^{\prime}(t)-1\right)$. We also remark that (21) implies that (22) is satisfied on $(-\infty, 0)$ and on $(0,+\infty)$.

Let us denote the expression at (22) by $g(t)$. By (22) $\lim _{t \rightarrow 0} g(t)$ is a finite positive real number, and this assumption is equivalent to (23). Since the other 
claims of Remark 13 are not going to be used in our proof of Theorem 4, we just indicate their proofs briefly. If $f(t)$ is a convex function on $(0, \infty)$, then $t f(1 / t)$ is also convex (which follows by differentiation and the inequality

$$
f\left(\frac{1}{t_{1}}\right)-f\left(\frac{1}{t_{2}}\right) \leq\left(\frac{1}{t_{1}}-\frac{1}{t_{2}}\right) f^{\prime}\left(\frac{1}{t_{1}}\right) \leq \frac{1}{t_{1}} f^{\prime}\left(\frac{1}{t_{1}}\right)-\frac{1}{t_{2}} f^{\prime}\left(\frac{1}{t_{2}}\right),
$$

where $0<t_{1}<t_{2}$.) This implies the last claim of Remark 13. So for the first claim we just have to observe that $\lim _{t \rightarrow 0^{-}} g^{\prime}(t) \leq \lim _{t \rightarrow 0^{+}} g^{\prime}(t)$ is equivalent to $\lim _{t \rightarrow+\infty}$ $t\left(t Q^{\prime}(t)-1\right) \leq \lim _{t \rightarrow-\infty} t\left(t Q^{\prime}(t)-1\right)$. This can easily be done by calculating $g^{\prime}(t)$ and then using (23).

Some consequences of (21) and (22) are as follows:

$$
\lim _{t \rightarrow \pm \infty}|t| W(t)=\rho \in(0,+\infty) \text { exists. }
$$

Since $\exp (Q(t))$ is convex, $\exp (Q(t))$ is absolutely continuous inside $\mathbb{R}$ which implies that both $W(t)$ and $Q(t)$ are absolutely continuous inside $\mathbb{R}$.

$Q^{\prime}(t)$ is bounded inside $\mathbb{R}$ a.e. because by (21) $\exp (Q(t)) Q^{\prime}(t)$ is increasing a.e.

We collected below some frequently used definitions and notation in the paper.

Definitions 14. Let $L \subset \mathbb{R}$ and let $f: L \rightarrow \mathbb{R} \cup\{-\infty\} \cup\{+\infty\}$.

$f$ is Hölder continuous with Hölder index $0<\tau \leq 1$ if with some $K$ constant $|f(x)-f(y)| \leq K|x-y|^{\tau}, x, y \in L$. In this case we write $f \in H^{\tau}(L)$.

The $L^{p}$ norm of $f$ is denoted by $\|f\|_{p}$. When $p=\infty$ we will also use the $\|f\|_{L}$ notation.

We say that an integral or limit exists if it exists as a real number.

Let $x \in \mathbb{R}$. If $f$ is integrable on $L \backslash(x-\epsilon, x+\epsilon)$ for all $0<\epsilon$, then the Cauchy principal value integral is defined as

$$
P V \int_{L} f(t) d t:=\lim _{\epsilon \rightarrow 0^{+}} \int_{L \backslash(x-\epsilon, x+\epsilon)} f(t) d t,
$$

if the limit exists.

It is known that $P V \int_{L} g(t) /(t-x) d t$ exists for almost every $x \in \mathbb{R}$ if $g: L \rightarrow \mathbb{R}$ is integrable.

For $0<\iota$ and $a \in \mathbb{R}$ we define

$$
a_{\iota}^{+}:=\max (a, \iota) \text { and } a_{\iota}^{-}:=\max (-a, \iota) .
$$

For $a>b$ the interval $[a, b]$ is an empty set.

We say that a property is satisfied inside $L$ if it is satisfied on all compact subsets of $L$.

$o(1)$ will denote a quantity which is approaching zero. For example, we may write $10^{x}=100+o(1)$ as $x \rightarrow 2$. Sometimes we also specify the domain (which may change with $\epsilon$ ) where the equation should be considered. For example, $\sin (x)=o(1)$ for $x \in[\pi, \pi+\epsilon]$ when $\epsilon \rightarrow 0^{+}$.

Recall that $S_{W^{\lambda}}$ denotes the support of the equilibrium measure $\mu_{W^{\lambda}}$. Since it is an interval (see Lemma 16), let $\left[a_{\lambda}, b_{\lambda}\right]:=S_{W^{\lambda}}$.

For $x \notin\left(a_{\lambda}, b_{\lambda}\right)$ let $V_{\lambda}(x):=0$, and for a.e. $x \in\left(a_{\lambda}, b_{\lambda}\right)$ let

$$
V_{\lambda}(x):=\frac{P V \int_{a_{\lambda}}^{b_{\lambda}} \frac{\lambda \sqrt{\left(t-a_{\lambda}\right)\left(b_{\lambda}-t\right)} Q^{\prime}(t)}{t-x} d t}{\pi^{2} \sqrt{\left(x-a_{\lambda}\right)\left(b_{\lambda}-x\right)}}+\frac{1}{\pi \sqrt{\left(x-a_{\lambda}\right)\left(b_{\lambda}-x\right)}} .
$$


Let $x \in[-1,1]$. Depending on the value of $c \in[-1,1]$ the following integrals may or may not be principal value integrals:

$$
\begin{gathered}
v_{c}(x):=-P V \int_{-1}^{c} \frac{\lambda \sqrt{1-t^{2}} e^{-Q(t)}}{\pi^{2} \sqrt{1-x^{2}}(t-x)} d t, \\
h_{c}(x):=P V \int_{c}^{1} \frac{\lambda \sqrt{1-t^{2}} e^{-Q(t)}}{\pi^{2} \sqrt{1-x^{2}}(t-x)} d t .
\end{gathered}
$$

(We should keep it in mind that $v_{c}(x)$ and $h_{c}(x)$ also depend on $\lambda$.)

Define

$B(x):=v_{c}(x)-h_{c}(x)=v_{1}(x)=-P V \int_{-1}^{1} \frac{\lambda \sqrt{1-t^{2}} e^{-Q(t)}}{\pi^{2} \sqrt{1-x^{2}}(t-x)} d t, \quad x \in[-1,1]$.

$P_{n}(x)$ and $p_{n}(x)$ will denote polynomials of degree at most $n$.

Remark 15. Since $\exp (-Q)$ is absolutely continuous inside $\mathbb{R}$ and $(\exp (-Q))^{\prime}=$ $-\exp (-Q) Q^{\prime}$ is bounded a.e. on $[-1,1]$, by the fundamental theorem of calculus we see that $\exp (-Q(t)) \in H^{1}([-1,1])$. Also, $\sqrt{1-t}, \sqrt{1+t} \in H^{0.5}([-1,1])$, so $\sqrt{1-t} \sqrt{1+t} \exp (-Q(t)) \in H^{0.5}([-1,1])$, so $\sqrt{1-x^{2}} B(x) \in H^{0.5}([-1,1])$ by the Plemelj-Privalov Theorem $([6], \S 19)$. As a consequence, $v_{c}(x)$ and $h_{c}(x)$ exist for any $x \in[-1,1] \backslash\{c\}$.

Let $[a, b]$ be an arbitrary interval. Our plan is to apply Theorem 11 with $w=W^{\lambda}$, where $\lambda>1$ and $\lambda$ is sufficiently close to 1 . Hence we need to show that the conditions of the theorem are fulfilled.

Lemma 16. Let $1<\lambda$. Then $S_{W^{\lambda}}$ is a finite interval $\left[a_{\lambda}, b_{\lambda}\right]$, and $\mu_{W^{\lambda}}$ is absolutely continuous with respect to the Lebesgue measure. Also, its density is $d \mu_{W^{\lambda}}(x)=$ $V_{\lambda}(x) d x$.

Proof. Let $1<p$. Note that $\exp (\lambda Q(x))$ is a convex function because it is the composition of the positive convex $\exp (Q(x))$ function and the increasing convex $x^{\lambda}, 0 \leq x$, function. So by [2], Theorem $5, S_{W^{\lambda}}$ is an interval $\left[a_{\lambda}, b_{\lambda}\right]$, which is finite since $\lim _{x \rightarrow \pm \infty}|x| W^{\lambda}(x)=0$. The density function $\left(d \mu_{W^{\lambda}}(x)\right) / d x$ exists since $\left(W^{\lambda}\right)^{\prime}=-\exp (-\lambda Q) \lambda Q^{\prime} \in L^{p}(\mathbb{R})$; see Theorem IV.2.2 of $[8$.

The integral at (24) is the Hilbert transform on $\mathbb{R}$ of the function defined as $\lambda \sqrt{\left(t-a_{\lambda}\right)\left(b_{\lambda}-t\right)} Q^{\prime}(t)$ on $\left(a_{\lambda}, b_{\lambda}\right)$ and 0 elsewhere. This function is in $L^{p}(\mathbb{R})$, so by the M. Riesz theorem the integral is also in $L^{p}(\mathbb{R})$; hence $V_{\lambda}(x)$ exists for a.e. $x \in\left[a_{\lambda}, b_{\lambda}\right]$. Moreover, by the Hölder inequality $(1 / a+1 / b=1 / c$ implies $\left.\|f g\|_{c} \leq\|f\|_{a}\|g\|_{b}\right)$ we see that $V_{\lambda} \in L_{1.9}(\mathbb{R})$, so $V_{\lambda} \in L_{1}(\mathbb{R})$, too.

By the proof of Lemma 16 of [1, the function $V_{\lambda}$ satisfies $\int V_{\lambda}(x) d x=1$ and

$$
\int_{a_{\lambda}}^{b_{\lambda}} \log |t-x| V_{\lambda}(t) d t=\lambda Q(x)+C, \quad x \in\left(a_{\lambda}, b_{\lambda}\right) .
$$

The left hand side is well defined since by the Hölder inequality

$$
x \mapsto \int_{a_{\lambda}}^{b_{\lambda}}|\log | t-x||\left|V_{\lambda}(t)\right| d t \quad \text { is uniformly bounded on }\left[a_{\lambda}, b_{\lambda}\right] .
$$

Consider the unit signed measure $\mu$ defined by $d \mu(x):=V_{\lambda}(x) d x$. By (25) $U^{\mu}(x)+\lambda Q(x)=-C, x \in\left(a_{\lambda}, b_{\lambda}\right)$. From this and from $U^{\mu_{W^{\lambda}}}(x)+\lambda Q(x)=F_{W^{\lambda}}$, 
$x \in\left[a_{\lambda}, b_{\lambda}\right]$, we get $U^{\mu}(x)=U^{\mu_{W^{\lambda}}}(x)+C_{2}, x \in\left(a_{\lambda}, b_{\lambda}\right)$. But (26) shows that $U^{\mu^{+}}(x)$ and $U^{\mu^{-}}(x)$ are finite for all $x \in\left[a_{\lambda}, b_{\lambda}\right]$. So $U^{\mu^{+}}(x)=U^{\mu_{W^{\lambda}}+\mu^{-}}(x)+C_{2}$, $x \in\left(a_{\lambda}, b_{\lambda}\right)$. Here $\mu^{+}$and $\mu_{W^{\lambda}}+\mu^{-}$are positive measures which have the same mass. $\mu_{W^{\lambda}}, \mu^{-}$(and $\mu^{+}$) all have finite logarithmic energy (see (26)), hence $\mu_{W^{\lambda}}+$ $\mu^{-}$has it, too. Applying Theorem II.3.2 of 8 w we get $U^{\mu^{+}}(z)=U^{\mu_{W^{\lambda}}+\mu^{-}}(z)+C_{2}$ for all $z \in \mathbb{C}$. By the unicity theorem ([8], Theorem II.2.1) $\mu^{+}=\mu_{W^{\lambda}}+\mu^{-}$. Hence $\mu=\mu_{W^{\lambda}}$ and our lemma is proved.

Lemma 17. For any $[a, b]$ interval, if $1<\lambda$ and $\lambda$ is sufficiently close to 1 , then $[a, b] \subset\left(a_{\lambda}, b_{\lambda}\right)$ and $V_{\lambda}(x)$ has positive lower bound a.e. on $[a, b]$.

Proof. First we show that $\lim _{\lambda \rightarrow 1^{+}} a_{\lambda}=-\infty$ and $\lim _{\lambda \rightarrow 1^{+}} b_{\lambda}=+\infty$. Fix $z \in \mathbb{R}$ and let let $\lambda_{n} \searrow 1$ be arbitrary. We show that $z \in\left(a_{\lambda_{n}}, b_{\lambda_{n}}\right)$ for large $n$. If this were not the case, then for a subsequence (indexed also by $\lambda_{n}$ ) we have

$$
\left[a_{\lambda_{n}}, b_{\lambda_{n}}\right] \subset[z,+\infty)
$$

(Or, for a subsequence we have $\left[a_{\lambda_{n}}, b_{\lambda_{n}}\right] \subset(-\infty, z]$, which can be handled similarly.) $\overline{\mathbb{R}}$ is compact, so by Helly's Selection Theorem ([8, Theorem 0.1.3) we can find a subsequence of the equilibrium measures $\mu_{W^{\lambda_{n}}}$ (indexed also by $\lambda_{n}$ ) which weak-* converges to a probability measure $\mu$. This we denote by $\mu_{W^{\lambda_{n}}} \stackrel{*}{\rightarrow} \mu$.

For fixed large $0<N$ we define the probability measure

$$
\nu_{N}:=\frac{\left.\mu_{W}\right|_{[-N, N]}}{\left\|\left.\mu_{W}\right|_{[-N, N]}\right\|} .
$$

We remark that $\mu_{W}(\{\infty\})=0$, which implies that

$$
\left.|| \mu_{W}\right|_{[-N, N]} \| \rightarrow 1 \text { as } N \rightarrow+\infty .
$$

By $([9$, p. 3$)$ there exists $K \in \mathbb{R}$ such that

$$
K \leq \log \frac{1}{|z-t| W(z) W(t)}, \quad z, t \in \overline{\mathbb{R}} .
$$

Now we show that

$$
\iint \log \frac{1}{|z-t| W^{\lambda_{1}}(z) W^{\lambda_{1}}(t)} d \nu_{N}(t) d \nu_{N}(z) \text { is finite. }
$$

By (29) the double integral at (30) is bounded from below. It equals

$$
\iint \log \frac{1}{|z-t|^{\lambda_{1}} W^{\lambda_{1}}(z) W^{\lambda_{1}}(t)} d \nu_{N}(t) d \nu_{N}(z)+\iint \log |z-t|^{\lambda_{1}-1} d \nu_{N}(t) d \nu_{N}(z) .
$$

Here the first double integral is finite because $V_{W}$ is finite ([9], Theorem 1.2). Also, the second integral is bounded from above since $\nu_{N}$ has compact support. So (30) is established. 
Choose $0<\tau$ such that $\|\tau W(x)\|_{\infty} \leq 1$. Now,

$$
\begin{gathered}
I_{W}(\mu)-\log \left(\tau^{2}\right)=\lim _{M \rightarrow+\infty} \iint \min \left(M, \log \frac{1}{|z-t|(\tau W(z))(\tau W(t))}\right) d \mu(t) d \mu(z) \\
=\lim _{M \rightarrow+\infty} \lim _{n \rightarrow+\infty} \iint \min \left(M, \log \frac{1}{|z-t|(\tau W(z))(\tau W(t))}\right) d \mu_{W^{\lambda_{n}}}(t) d \mu_{W^{\lambda_{n}}}(z) \\
\leq \lim _{n \rightarrow+\infty} \iint \log \frac{1}{|z-t|(\tau W(z))^{\lambda_{n}}(\tau W(t))^{\lambda_{n}}} d \mu_{W^{\lambda_{n}}}(t) d \mu_{W^{\lambda_{n}}}(z) \\
\leq \lim _{n \rightarrow+\infty} \iint \log \frac{1}{|z-t|(\tau W(z))^{\lambda_{n}}(\tau W(t))^{\lambda_{n}}} d \nu_{N}(t) d \nu_{N}(z) \\
\quad=\iint \log \frac{1}{|z-t| W(z) W(t)} d \nu_{N}(t) d \nu_{N}(z)-\log \left(\tau^{2}\right) .
\end{gathered}
$$

In the first equality above we used the monotone convergence theorem (see also (29)). In the second equality we used $\mu_{W^{\lambda_{n}}} \times \mu_{W^{\lambda_{n}}} \stackrel{*}{\rightarrow} \mu \times \mu$. In the second inequality it was used that $\mu_{W^{\lambda_{n}}}$ is the probability measure which minimizes the double integral of $-\log \left(|z-t| W^{\lambda_{n}}(z) W^{\lambda_{n}}(t)\right)$. In the last equality we used the monotone convergence theorem again. (It can be used because of (29), plus the integral is finite even with the power $\lambda_{1}$ by (30).)

Also,

$$
\begin{gathered}
\iint\left[\log \frac{1}{|z-t| W(z) W(t)}-K\right] d \nu_{N}(t) d \nu_{N}(z) \\
\leq \iint\left[\log \frac{1}{|z-t| W(z) W(t)}-K\right] \frac{d \mu_{W}(t)}{\|\left.\mu_{W}\right|_{[-N, N]}||} \frac{d \mu_{W}(z)}{\left\|\left.\mu_{W}\right|_{[-N, N]}\right\|} .
\end{gathered}
$$

Combining this with (31) we have

$$
I_{W}(\mu) \leq K\left[1-\frac{1}{\|\left.\mu_{W}\right|_{[-N, N]}||^{2}}\right]+\frac{1}{\left\|\left.\mu_{W}\right|_{[-N, N]}\right\|^{2}} V_{W} .
$$

Letting $N \rightarrow+\infty$ we gain $I_{W}(\mu) \leq V_{W}$. Therefore $\mu=\mu_{W}$. Thus $\mu_{W^{\lambda_{n}}} \stackrel{*}{\rightarrow} \mu_{W}$ which contradicts (27), since $S_{W}=\overline{\mathbb{R}}$ (see Lemma 25).

To prove the positive lower bound of $V_{\lambda}(x)$ a.e. on $[a, b]$, let $I:=[a-1, b+1]$. Since $W^{\lambda}$ is an admissible weight, we can use [8], Theorem IV.4.9, to get

$$
\left.\mu_{W^{\lambda}}\right|_{S_{W^{\lambda^{2}}}} \geq\left.\left(1-\frac{1}{\lambda^{2}}\right) \omega_{S_{W \lambda}}\right|_{S_{W^{\lambda^{2}}}}
$$

where $\omega_{S_{W^{\lambda}}}$ is the classical equilibrium measure of the set $S_{W^{\lambda}}$ (with no external field present). (We remark that $S_{W^{\lambda}} \supset S_{W^{\lambda^{2}}}$.)

It follows that if $\lambda$ is so close to 1 that $S_{W^{\lambda^{2}}} \supset I$ holds, then $[a, b] \subset\left(a_{\lambda}, b_{\lambda}\right)$ and $V_{\lambda}(x)$ has positive lower bound a.e. on $[a, b]$.

Our next goal is to show that $V_{\lambda}(x)$ has a smooth integral on $[a, b]$, if $\lambda>1$ and $\lambda$ is sufficiently close to 1 . To this end we need some lemmas. First, we will need Lemma 22 of [1]. We formulate it as follows. 
Lemma 18. Let $A<B<1, f \in L^{1}[A, 1]$ and $f \in H^{1}[A,(B+1) / 2]$. Define $v^{*}(x):=\int_{c}^{1} f(t) /(t-x) d t$, where $c \in[A, B]$ and $x<c$. Then

$$
v^{*}(x)=(f(c)+o(1)) \log \frac{1}{c-x}, \quad \text { as } \quad x \rightarrow c^{-} .
$$

Here $o(1)$ depends on $c-x$ only.

Lemma 19. Let $-1<a<b<1$ and $0<\iota$ be fixed. Let $0<\epsilon<1 / 10$ and $\delta:=\sqrt{\epsilon}-2 \epsilon$. Then for $x_{1}, x_{2} \in[a, b] \cap(c-\delta, c+\delta)^{c},\left|x_{1}-x_{2}\right| \leq \epsilon$, all the quotients

$$
\frac{v_{c}\left(x_{1}\right)_{\iota}^{+}}{v_{c}\left(x_{2}\right)_{\iota}^{+}}, \quad \frac{v_{c}\left(x_{1}\right)_{\iota}^{-}}{v_{c}\left(x_{2}\right)_{\iota}^{-}}, \quad \frac{h_{c}\left(x_{1}\right)_{\iota}^{+}}{h_{c}\left(x_{2}\right)_{\iota}^{+}}, \frac{h_{c}\left(x_{1}\right)_{\iota}^{-}}{h_{c}\left(x_{2}\right)_{\iota}^{-}}
$$

are equal to $1+o(1)$ as $\epsilon \rightarrow 0^{+}$. Here the o(1) term is independent of $x_{1}, x_{2}$ and $c$.

Proof. First we consider the case when $x_{1}, x_{2} \leq c-\delta$. Note that for $x_{1}>x_{2}$ we have $1 /\left(t-x_{2}\right)<1 /\left(t-x_{1}\right), t \in[c, 1]$, whereas for $x_{1} \leq x_{2}$ we have

$$
\frac{1}{t-x_{2}} \leq\left(1+\frac{x_{2}-x_{1}}{c-x_{2}}\right) \frac{1}{t-x_{1}}=(1+o(1)) \frac{1}{t-x_{1}}, \quad t \in[c, 1] .
$$

Multiplying these inequalities by $\lambda \sqrt{1-t^{2}} \exp (-Q(t)) / \pi^{2}$ and integrating on $[c, 1]$ we gain

$$
\frac{h_{c}\left(x_{2}\right)}{h_{c}\left(x_{1}\right)}=1+o(1)
$$

where $\sqrt{1-x_{2}^{2}} / \sqrt{1-x_{1}^{2}}=1+o(1)$ was also used. By the same argument, if $x_{1}, x_{2} \geq c+\delta$, we have $v_{c}\left(x_{2}\right) / v_{c}\left(x_{1}\right)=1+o(1)$, from which

$$
\frac{v_{c}\left(x_{2}\right)_{\iota}^{+}}{v_{c}\left(x_{1}\right)_{\iota}^{+}}=1+o(1) \text {. }
$$

Returning to the case of $x_{1}, x_{2} \leq c-\delta$, from $v_{c}(x)=h_{c}(x)+B(x)$, from (32) and from $B\left(x_{2}\right)=B\left(x_{1}\right)+o(1)$ we get

$$
\begin{aligned}
\mid v_{c}\left(x_{2}\right) & -v_{c}\left(x_{1}\right)|=| o(1) \mid\left(1+\left|v_{c}\left(x_{1}\right)-B\left(x_{1}\right)\right|\right) \\
& \leq|o(1)|\left(\left|v_{c}\left(x_{1}\right)\right|+1+\|B\|_{[a, b]}\right) .
\end{aligned}
$$

Assuming $\left|v_{c}\left(x_{1}\right)\right| \leq 1$, we have

$$
\left|v_{c}\left(x_{2}\right)_{\iota}^{+}-v_{c}\left(x_{1}\right)_{\iota}^{+}\right| \leq\left|v_{c}\left(x_{2}\right)-v_{c}\left(x_{1}\right)\right| \leq|o(1)|,
$$

so (33) holds again. Finally, if $\left|v_{c}\left(x_{1}\right)\right| \geq 1$, then from (34)

$$
\left|\frac{v_{c}\left(x_{2}\right)}{v_{c}\left(x_{1}\right)}-1\right|=|o(1)|\left(1+\frac{1+\|B\|_{[a, b]}}{\left|v_{c}\left(x_{1}\right)\right|}\right)=|o(1)|,
$$

from which (33) again easily follows.

The proof of the rest of our lemma is similar.

Lemma 20. Let $-1<a<b<1$ and $0<\iota$ be fixed. Then the family of functions $\mathcal{F}^{+}:=\left\{v_{c}(x)_{\iota}^{+}: c \in[-1,1]\right\}$ and $\mathcal{F}^{-}:=\left\{v_{c}(x)_{\iota}^{-}: c \in[-1,1]\right\}$ have uniformly smooth integrals on $[a, b]$. 
Proof. We consider $\mathcal{F}^{+}$only $\left(\mathcal{F}^{-}\right.$can be handled similarly). Let $c \in[-1,1]$. Let $I:=[u-\epsilon, u], J:=[u, u+\epsilon]$ be two adjacent intervals of $[a, b]$, where $0<\epsilon<1 / 10$. We have to show that

$$
\frac{\int_{I} v_{c}(t)_{\iota}^{+} d t}{\int_{J} v_{c}(t)_{\iota}^{+} d t}=1+o(1), \quad \text { as } \quad \epsilon \rightarrow 0^{+},
$$

where $o(1)$ is independent of $I, J$ and $c$. Let $0<\epsilon<1 / 10$ and let $\delta:=\sqrt{\epsilon}-2 \epsilon(>\epsilon)$.

Case 1: Assume $I \cup J \subset(c-\delta, c+\delta)^{c}$. From Lemma 19 we have $v_{c}(t)_{\iota}^{+}=$ $(1+o(1)) v_{c}(t+\epsilon)_{\iota}^{+}, t \in I$. Thus $\int_{I} v_{c}(t)_{\iota}^{+} d t=(1+o(1)) \int_{J} v_{c}(t)_{\iota}^{+} d t$.

Case 2: Assume $(I \cup J) \cap(c-\delta, c+\delta) \neq \emptyset$. So $I \cup J \subset[c-\sqrt{\epsilon}, c+\sqrt{\epsilon}]$. Let $\epsilon$ be so small that $c \in[(a-1) / 2,(b+1) / 2]$. (This can be done because of our assumption of Case 2.)

Let $f(t):=\lambda \sqrt{1-t^{2}} \exp (-Q(t)) / \pi^{2}$. Applying Lemma 18 (with $A:=(a-1) / 2$, $B:=(b+1) / 2)$ we have $\sqrt{1-x^{2}} h_{c}(x)=(f(c)+o(1))(-\log |c-x|)$ for $x \in[c-\sqrt{\epsilon}, c)$ as $\epsilon \rightarrow 0^{+}$, which easily leads to

$$
h_{c}(x)=\left(\frac{f(c)}{\sqrt{1-c^{2}}}+o(1)\right)(-\log |c-x|) \text { for } x \in[c-\sqrt{\epsilon}, c) \text { as } \epsilon \rightarrow 0^{+} .
$$

From here using $h_{c}(x)=v_{c}(x)-B(x)$ we get

$$
v_{c}(x)=\left(\frac{f(c)}{\sqrt{1-c^{2}}}+o(1)\right)(-\log |c-x|) \text { for } x \in[c-\sqrt{\epsilon}, c) \text { as } \epsilon \rightarrow 0^{+} .
$$

Clearly, (35) also holds for $x \in(c, c+\sqrt{\epsilon}$ (which can be seen by stating Lemma 18 for $-1<A<B$ instead of $A<B<1)$.

$f(x)$ has a positive lower bound on $[(a-1) / 2,(b+1) / 2]$. So we can choose $\epsilon$ so small that the right hand side of (35) is at least $\iota$ for all possible values of $c$ and $x$. Hence $v_{c}(x)=v_{c}(x)_{\iota}^{+}$and

$$
\frac{\int_{I} v_{c}(t)_{\iota}^{+} d t}{\int_{J} v_{c}(t)_{\iota}^{+} d t}=\frac{\left(\frac{f(c)}{\sqrt{1-c^{2}}}+o(1)\right) \int_{I} \log \frac{1}{|c-t|} d t}{\left(\frac{f(c)}{\sqrt{1-c^{2}}}+o(1)\right) \int_{J} \log \frac{1}{|c-t|} d t}=(1+o(1))^{2}=1+o(1)
$$

where we used the fact that $\log (1 /|x|)$ has a smooth integral on $[-1 / 2,1 / 2]([1]$, Proposition 20).

Lemma 21. Let $F(x)=G(x)-H(x)$, where $F(x), G(x), H(x)$ are a.e. nonnegative functions defined on an interval, $G(x)$ and $H(x)$ have smooth integrals and $H(x) \leq(1-\eta) G(x)$ a.e. with some $\eta \in(0,1)$. Then $F(x)$ has a smooth integral.

Proof. Let $I$ and $J$ be two adjacent intervals of equal lengths $\epsilon$, where $\epsilon$ is "small enough". Let $a:=\int_{I} G, A:=\int_{J} G, b:=\int_{I} H, B:=\int_{J} H$. By assumption

$$
A=(1+o(1)) a \quad \text { and } \quad B=(1+o(1)) b, \quad \text { as } \epsilon \rightarrow 0^{+},
$$

and we have to show that $A-B=(1+o(1))(a-b)$.

We may assume that $a-b \neq 0$, otherwise $F(x)=0$ a.e. on $I$ which implies $a=b=0$ and so $A=B=0$.

Integrating $H \leq(1-\eta) G$ on $I$ we get $b \leq(1-\eta) a$, from which $(a+b) /$ $(a-b) \leq(1+(1-\eta)) /(1-(1-\eta))$. Thus, from (36)

$$
|(A-a)-(B-b)| \leq|o(1)|(a+b) \leq|o(1)|(a-b) .
$$


Following the proof of Lemma 24 of [1] we will prove the following lemma. But we remark that the absolutely continuous hypothesis of Lemma 24 is unnecessary in [1].

Lemma 22. Let $N(x)$ be a bounded, increasing, right-continuous function on $[-1,1]$ and let $f(x) \in L^{1}([-1,1])$ be nonnegative. Then

$$
P V \int_{-1}^{1} \frac{f(t) N(t)}{t-x} d t=-N(1) f_{1}(x)+\int_{(-1,1]} f_{t}(x) d N(t), \quad \text { a.e. } x \in[-1,1],
$$

where the integral on the right hand side is a Lebesgue-Stieltjes integral and

$$
f_{c}(x):=-P V \int_{-1}^{c} \frac{f(t)}{t-x} d t, \quad \text { a.e. } x \in[-1,1] .
$$

Proof. Let us denote the left hand side of (37) by $F(x)$. Since $f(x)$ and $f(x) N(x)$ are in $L^{1}[-1,1]$ and $N(x)$ is increasing, there is a set of full measure in $(-1,1)$ where $f_{1}(x), F(x)$ and $N^{\prime}(x)$ all exist. Let $x$ be chosen from this set. It follows that $f_{c}(x)$ exist for all $c \in[-1,1] \backslash\{x\}$. Also,

$$
F(x)=\lim _{\epsilon \rightarrow 0^{+}}\left(\int_{-1}^{x-\epsilon} \frac{f(t) N(t)}{t-x} d t+\int_{x+\epsilon}^{1} \frac{f(t) N(t)}{t-x} d t\right) .
$$

$t \rightarrow f_{t}(x)$ is an absolute continuous increasing function inside $[-1, x)$ and is an absolute continuous decreasing function inside $(x, 1]$, so at (38) we can use integration by parts to get

$$
\begin{gathered}
\int_{-1}^{x-\epsilon}+\int_{x+\epsilon}^{1}=-f_{x-\epsilon}(x) N(x-\epsilon)+f_{-1}(x) N(-1)+\int_{(-1, x-\epsilon]} f_{t}(x) d N(t) \\
+f_{x+\epsilon}(x) N(x+\epsilon)-f_{1}(x) N(1)+\int_{(x+\epsilon, 1]} f_{t}(x) d N(t) .
\end{gathered}
$$

But above $f_{-1}(x)=0$ and

$$
\begin{gathered}
f_{x+\epsilon}(x) N(x+\epsilon)-f_{x-\epsilon}(x) N(x-\epsilon) \\
=\left[f_{x+\epsilon}(x)-f_{x-\epsilon}(x)\right] N(x+\epsilon)+f_{x-\epsilon}(x)[N(x+\epsilon)-N(x-\epsilon)] .
\end{gathered}
$$

Note that

$$
f_{x+\epsilon}(x)-f_{x-\epsilon}(x)=-P V \int_{x-\epsilon}^{x+\epsilon} \frac{f(t)}{t-x} d t \rightarrow 0 \quad \text { as } \epsilon \rightarrow 0^{+},
$$

since $f_{1}(x)$ exists.

We claim that $\epsilon f_{x-\epsilon}(x) \rightarrow 0$ (and so the second term on the right hand side of (39) also tends to 0 since $N$ is differentiable at $x$ ). In other words we claim that

$$
\lim _{\epsilon \rightarrow 0^{+}} \epsilon \int_{\epsilon}^{1} \frac{g(t)}{t} d t \rightarrow 0
$$

for any integrable nonnegative $g(t)$ function. Integration by parts easily yields to

$$
\begin{gathered}
\lim _{\epsilon \rightarrow 0^{+}} \epsilon \int_{\epsilon}^{1} \frac{g(t)}{t} d t=\lim _{\epsilon \rightarrow 0^{+}} \epsilon \int_{\epsilon}^{1} \frac{\int_{0}^{t} g(u) d u}{t^{2}} d t \\
=\lim _{\epsilon \rightarrow 0^{+}}\left(\epsilon \int_{\epsilon}^{\rho}\right)+\lim _{\epsilon \rightarrow 0^{+}}\left(\epsilon \int_{\rho}^{1}\right) \leq \int_{0}^{\rho} g(u) d u+\lim _{\epsilon \rightarrow 0^{+}}\left(\epsilon \int_{\rho}^{1}\right),
\end{gathered}
$$

where $\rho$ was chosen such that $\int_{0}^{\rho} g(u) d u$ is small. This verifies our claim. 
Putting these together, we get that on one hand,

$$
\lim _{\epsilon \rightarrow 0^{+}}\left(\int_{(-1, x-\epsilon]} f_{t}(x) d N(t)+\int_{(x+\epsilon, 1]} f_{t}(x) d N(t)\right)
$$

exists and equals $F(x)+f_{1}(x) N(1)$, and on the other hand, (40) equals to

$$
\int_{(-1,1] \backslash\{x\}} f_{t}(x) d N(t)=\int_{(-1,1]} f_{t}(x) d N(t)
$$

by the monotone convergence theorem (which can be used since $c \rightarrow f_{c}(x)$ is bounded from below on $[-1,1]$ since $f_{1}(x)$ is finite). The continuity of $N$ at $x$ allowed us to integrate on the whole $(-1,1]$ at (41)).

Lemma 23. Let $[a, b]$ be arbitrary and let $1<\lambda$ be chosen to satisfy the conclusion of Lemma 17. Then $V_{\lambda}(x)$ has a smooth integral on $[a, b]$.

Proof. To keep the notation simple we will assume that $-1<a<b<1$, and $a_{\lambda}=-1, b_{\lambda}=1$, that is, the support of $\mu_{W^{\lambda}}$ is $[-1,1]$. This can be done without loss of generality. Define

$$
v(t):=\frac{\lambda \sqrt{1-t^{2}} e^{-Q(t)}}{\pi^{2} \sqrt{1-x^{2}}} \text { and } M(t):=\lim _{s \rightarrow t^{+}} e^{Q(s)} Q^{\prime}(s),
$$

where $v(t)$ also depends on the choice of $x$. Note that $M(t), t \in[-1,1]$, is a bounded, increasing, right-continuous function which agrees with $\exp (Q(t)) Q^{\prime}(t)$ almost everywhere.

Applying Lemma 22 for $f(t):=v(t)$ and $N(t):=M(t)$, let us fix an $x \in[a, b]$ value for which both (37) and $d \mu_{W^{\lambda}}(x)=V_{\lambda}(x) d x$ are satisfied. (These are satisfied almost everywhere.) From (24) and Lemma 22 we have

$$
\begin{gathered}
V_{\lambda}(x)=\frac{1}{\pi \sqrt{1-x^{2}}}+P V \int_{-1}^{1} \frac{\lambda \sqrt{1-t^{2}} Q^{\prime}(t)}{\pi^{2} \sqrt{1-x^{2}}(t-x)} d t \\
=\frac{1}{\pi \sqrt{1-x^{2}}}+P V \int_{-1}^{1} \frac{v(t) M(t)}{t-x} d t=L(x)+\int_{(-1,1]} v_{t}(x) d M(t),
\end{gathered}
$$

where $L(x):=1 /\left(\pi \sqrt{1-x^{2}}\right)-M(1) B(x)$.

Let $0<\iota$. Since $L(x)$ is a continuous function on $[a, b]$ (see Remark [15), $L(x)_{\iota}^{+}$ and $L(x)_{\iota}^{-}$have smooth integrals on $[a, b]$.

Let $\mathcal{B}$ denote the Borel sets of $[-1,1]$ and let $\mathcal{L}$ denote the Lebesgue measurable sets of $[a, b]$. We claim that both $(t, x) \mapsto v_{t}(x)_{\iota}^{+}$and $(t, x) \mapsto v_{t}(x)_{\iota}^{-}$are $(\mathcal{B}, \mathcal{L})-$ measurable functions. Let $v_{c}(x)^{(\epsilon)}$ be defined the same way as $v_{c}(x)$, except we integrate on $[-1, c] \backslash(x-\epsilon, x+\epsilon)$ instead of $[-1, c]$. Then $(c, x) \mapsto v_{c}(x)^{(\epsilon)}$ is measurable for all $\epsilon>0$. Since $v_{c}(x)$ is the pointwise limit of $v_{c}(x)^{(\epsilon)}$ as $\epsilon \rightarrow 0$, we get that $(c, x) \mapsto v_{c}(x)$ is $(\mathcal{B}, \mathcal{L})$-measurable. From here our claim is clear.

By Lemma $20 \mathcal{F}^{+}$and $\mathcal{F}^{-}$have uniformly smooth integrals on $[a, b]$, so both

$$
\begin{gathered}
V_{\lambda}(x)_{(\iota)}^{(+)}:=L(x)_{\iota}^{+}+\int_{(-1,1]} v_{t}(x)_{\iota}^{+} d M(t) \quad \text { and } \\
V_{\lambda}(x)_{(\iota)}^{(-)}:=L(x)_{\iota}^{-}+\int_{(-1,1]} v_{t}(x)_{\iota}^{-} d M(t)
\end{gathered}
$$

have smooth integrals on $[a, b]$. (These new functions are not to be mixed with $V_{\lambda}(x)_{\iota}^{-}$and $\left.V_{\lambda}(x)_{\iota}^{-}.\right)$ 
Set

$$
V_{\lambda}(x)_{(\iota)}:=V_{\lambda}(x)_{(\iota)}^{(+)}-V_{\lambda}(x)_{(\iota)}^{(-)} .
$$

Then, using $\left|z_{\iota}^{+}-z_{\iota}^{-}-z\right| \leq \iota, z \in \mathbb{R}$, we get

$$
\begin{aligned}
\left|V_{\lambda}(x)_{(\iota)}-V_{\lambda}(x)\right| \leq & \left|L(x)_{\iota}^{+}-L(x)_{\iota}^{-}-L(x)\right| \\
& +\int_{(-1,1]}\left|v_{t}(x)_{\iota}^{+}-v_{t}(x)_{\iota}^{-}-v_{t}(x)\right| d M(t) \\
\leq & \iota+\int_{(-1,1]} \iota d M(t)=\iota(1+M(1)-M(-1)) .
\end{aligned}
$$

So

$$
V_{\lambda}(x)_{(\iota)} \rightarrow V_{\lambda}(x) \text { uniformly a.e. on }[a, b] \text { as } \iota \rightarrow 0^{+} .
$$

Also, since

$$
V_{\lambda}(x) \text { has positive lower bound a.e. on }[a, b] \text {, }
$$

$V_{\lambda}(x)_{(\iota)}$ has also positive lower bound a.e. on $[a, b]$, assuming $\iota$ is small enough. In addition, $v_{t}(x) \geq 0$ when $t \in[0, x]$, whereas $v_{t}(x) \geq B(x) \geq-\|B\|_{[a, b]}$ when $t \in(x, 1]$, so $V_{\lambda}(x)_{(\iota)}^{(-)}$is bounded a.e. on $[a, b]$. It follows that $V_{\lambda}(x)_{(\iota)}^{(-)} \leq$ $(1-\eta) V_{\lambda}(x)_{(\iota)}^{(+)}$a.e. $x \in[a, b]$ for some $\eta \in(0,1)$.

Applying Lemma 21] we conclude that $V_{\lambda}(x)_{(\iota)}$ has a smooth integral on $[a, b]$ (if $\iota$ is small enough). Therefore $V_{\lambda}(x)$ has a smooth integral by (43) and (44).

We now state Theorem 4 again and prove it.

Theorem 24. For a weight satisfying (21) and (22) we have $Z_{\overline{\mathbb{R}}}(W)=\emptyset$. That is, any continuous function $g: \overline{\mathbb{R}} \rightarrow \mathbb{R}$ can be uniformly approximated by weighted polynomials $W^{n} p_{n}(n=0,2,4, \ldots)$ on $\overline{\mathbb{R}}$.

Proof. Let $x_{0} \in \overline{\mathbb{R}}$. We show that $x_{0} \notin Z_{\overline{\mathbb{R}}}(W)$.

First let us assume that $x_{0}$ is finite. Choose $J:=[a, b]$ such that $a<x_{0}<b$ holds. Let $f(x)$ be a continuous function which is zero outside $J$ and $f\left(x_{0}\right) \neq 0$. Let $1<\lambda=u / v\left(u, v \in \mathbb{N}^{+}\right)$be a rational number for which the conclusion of Lemma 17 holds. Now we use Theorem [11, which is a powerful theorem of Totik. Since $V_{\lambda}$ has a positive lower bound a.e. on $J$ and it has a smooth integral on $J$ (see Lemma 23), by [11, Theorem 1.2, $(a, b) \cap Z\left(W^{\lambda}\right)=\emptyset$. So we can find $P_{n}$ $(n=0,1,2, \ldots)$ such that $\left(W^{\lambda}\right)^{n} P_{n} \rightarrow f$ uniformly on $\overline{\mathbb{R}}$.

So for $n:=N v$, we have

$$
W^{N u} p_{N u} \rightarrow f, \quad N=0,1,2, \ldots, \quad \text { uniformly on } \overline{\mathbb{R}},
$$

where $p_{N u}:=P_{N v}$ and $\operatorname{deg}\left(p_{N u}\right) \leq N v \leq N u$. For all fixed $s \in\{0, \ldots, u-1\}$ if we approximate $f / W^{s}$ instead of $f$ at (45), it easily follows that there exist $p_{k}$ $(k=0,1,2, \ldots)$ such that

$$
W^{k} p_{k} \rightarrow f, \quad k=0,1,2, \ldots, \quad \text { uniformly on } \overline{\mathbb{R}} \text {. }
$$

Using only $k=0,2,4, \ldots$, we get $x_{0} \notin Z_{\overline{\mathbb{R}}}(W)$ by Lemma 9 ,

Now let $x_{0}=\infty$. Define

$$
W_{0}(x):=\frac{1}{|x|} W\left(-\frac{1}{x}\right) .
$$


Note that $1 / W_{0}(x)(=|x| / W(-1 / x))$ and $|x| / W_{0}(-1 / x)(=1 / W(x))$ are positive and convex functions because $W$ satisfies (22) and (21).

Let $g$ be a continuous function on $\overline{\mathbb{R}}$. Define $-1 / \infty$ to be 0 and $-1 / 0$ to be $\infty$. (So $g(x)$ is continuous on $\overline{\mathbb{R}}$ if and only if $g(-1 / x)$ is continuous on $\overline{\mathbb{R}}$.) Observe that for some $p_{n}$ we have

$W^{n}(x) p_{n}(x) \rightarrow g(x)(n=0,2,4, \ldots)$ uniformly on $\overline{\mathbb{R}}$, iff

$W^{n}(-1 / x) p_{n}(-1 / x) \rightarrow g(-1 / x)(n=0,2,4, \ldots)$ uniformly on $\overline{\mathbb{R}}$, iff

$W_{0}{ }^{n}(x) q_{n}(x), \rightarrow g(-1 / x)(n=0,2,4, \ldots)$ uniformly on $\overline{\mathbb{R}}$,

where $q_{n}(x):=x^{n} p_{n}(-1 / x)$ are polynomials, $\operatorname{deg} q_{n} \leq n$.

Now let $f(x)$ be a continuous function on $\overline{\mathbb{R}}$ which is zero in a neighborhood of 0 but $f(\infty) \neq 0$. By what we have already proved, $q_{n}$ polynomials exist such that $W_{0}{ }^{n}(x) q_{n}(x)(n=0,2,4, \ldots)$ tends to $f(-1 / x)$ uniformly. Therefore we can approximate $f(x)$ uniformly by $W^{n}(x) p_{n}(x)(n=0,2,4, \ldots)$, where $p_{n}(x):=x^{n} q_{n}(-1 / x)$.

Now we establish the connection between homogeneous polynomials and weighted polynomials.

Let $B d(K)$ be the boundary of a two dimensional convex region $K \subset \mathbb{R}^{2}$ which is centrally symmetric to the origin $(0,0)$. For $t \in \mathbb{R}$ let $(x(t), y(t))$ be any of the two points on $B d(K)$ for which

$$
\frac{y(t)}{x(t)}=t
$$

Let $x(\infty):=0$ and choose the value $y(\infty)$ such that $(0, y(\infty)) \in B d(K)$. We define $y(\infty) / 0$ to be $\infty$, so (47) also holds for $t=\infty$.

Define

$$
W(t):=e^{-Q(t)}:=|x(t)|, \quad t \in \overline{\mathbb{R}} .
$$

Lemma 25. $W(t)$ satisfies properties (21), (22), and $S_{W}=\overline{\mathbb{R}}$.

Proof. $W$ is positive on $\mathbb{R}$.

We may assume that $x(t)>0, t \in \mathbb{R}$. Let $t_{1}, t_{3} \in \mathbb{R}$ and $t_{2}:=\alpha t_{1}+$ $(1-\alpha) t_{3}$, where $0<\alpha<1$. Let $\left(x_{2}, y_{2}\right)$ be the intersection of the line segments $\overline{\left(x\left(t_{1}\right), y\left(t_{1}\right)\right)\left(x\left(t_{3}\right), y\left(t_{3}\right)\right)}$ and $\overline{(0,0)\left(x\left(t_{2}\right), y\left(t_{2}\right)\right)}$. Note that $1 / x\left(t_{2}\right) \leq 1 / x_{2}$, and by elementary calculations

$$
\frac{1}{x_{2}}=\alpha \frac{1}{x\left(t_{1}\right)}+(1-\alpha) \frac{1}{x\left(t_{3}\right)},
$$

so (21) holds. The proof of (22) is identical to the proof of (21) once we notice that $y(-1 / t) / x(-1 / t)=-1 / t$, and so $|t| / W(-1 / t)=1 /|y(-1 / t)|$.

Now we show that $S_{W}=\overline{\mathbb{R}}$. Let $x$ be on the unit circle. Then $i(1+x) /(1-x) \in \overline{\mathbb{R}}$. Define the weight $w(x)$ by

$$
w(x)=\frac{1}{|1-x|} W\left(\frac{1+x}{1-x} i\right), \quad|x|=1 .
$$

(w(1) is defined by taking the limit of the right hand side as $x \rightarrow 1$.) One can study the weighted energy problem on the circle with weight $w(x)$ and the weighted energy problem on $\overline{\mathbb{R}}$ with weight $W(X)$, and observe that $S_{W}=\overline{\mathbb{R}}$ if and only if the support of the equilibrium measure on the circle is the full circle (see [3]). Note that $q(\theta):=-\log w\left(e^{i \theta}\right)=Q(-\cot (\theta / 2))+\log |\sin (\theta / 2)|+\log 2$ is continuous on $[0,2 \pi]$ by (23). It is also absolute continuous inside $(0,2 \pi)$, so it is absolute continuous on 
$[0,2 \pi]$. (We needed to show this in order to be able to apply Corollary 2.2 of [3].) Next we show that

$$
e^{q(\theta)}\left[2 \sin \left(\frac{\theta-c}{2}\right) q^{\prime}(\theta)-\cos \left(\frac{\theta-c}{2}\right)\right] \operatorname{sgn}\left(\sin \left(\frac{\theta-c}{2}\right)\right)
$$

is increasing on $(0,2 \pi)$ if $c:=0$, and it is increasing on $(\pi, 3 \pi)$ if $c:=\pi$. The former statement follows from (21) since if $c=0$, then on $(0,2 \pi)$ (48) equals $2 e^{Q(X)} Q^{\prime}(X)$, where $X:=i(1+x) /(1-x)$ (see (3.9) in [3]). The other statement can be seen similarly, by using (22). Now using Corollary 2.2 of [3], we gain that the support of the equilibrium measure on the circle is the full circle. Thus $S_{W}=\overline{\mathbb{R}}$.

Lemma 26. Let $f(x, y),(x, y) \in B d(K)$, be a continuous function such that $f(x, y)=f(-x,-y)$ for all $(x, y) \in B d(K)$. Then homogeneous polynomials

$$
h_{n}(x, y):=\sum_{k=0}^{n} a_{k}^{(n)} x^{n-k} y^{k}, \quad n=0,2,4, \ldots,
$$

exist such that $h_{n}(x, y) \rightarrow f(x, y)(n=0,2,4, \ldots)$ uniformly on $B d(K)$.

Proof. Recall the definition: $y(t) / x(t)=t, t \in \overline{\mathbb{R}}$, where $(x(t), y(t)) \in B d(K)$ and $W(t):=|x(t)|$. Define

$$
f(t):=f(x(t), y(t))=f(-x(t),-y(t)), \quad t \in \overline{\mathbb{R}} .
$$

Note that if $n$ is an even number (and $a_{k}^{(n)}$ are unknowns), then

$$
\begin{gathered}
\sum_{k=0}^{n} a_{k}^{(n)} x^{n-k}(t) y^{k}(t)=x^{n}(t) \sum_{k=0}^{n} a_{k}^{(n)}\left(\frac{y(t)}{x(t)}\right)^{k} \\
=|x(t)|^{n} \sum_{k=0}^{n} a_{k}^{(n)} t^{k}=W^{n}(t) p_{n}(t)
\end{gathered}
$$

where $p_{n}(t):=\sum_{k=0}^{n} a_{k}^{(n)} t^{k}, \operatorname{deg} p_{n} \leq n$. (When $t=\infty$, the left hand side of (49) again equals $\left.\left(W^{n} p_{n}\right)(\infty):=\lim _{t \rightarrow \pm \infty} W^{n}(t) p_{n}(t).\right)$

But by Theorem 24 there exist $W^{n}(t) p_{n}(t)(n=0,2,4, \ldots)$ which tend to $f(t)$ uniformly on $\overline{\mathbb{R}}$. This completes the proof, since for any $(x, y) \in B d(K)$ there exists $t \in \overline{\mathbb{R}}$ such that either $(x(t), y(t))=(x, y)$ or $(-x(t),-y(t))=(x, y)$.

Proof of Theorem 3. Define $f(x, y):=1,(x, y) \in B d(K)$. By Lemma 26 there exist $h_{2 n} \in H_{2 n}^{2}, n \in \mathbb{N}$, such that $\left\|1-h_{2 n}\right\|_{B d(K)} \rightarrow 0$. From here Theorem 3 follows the same way that Theorem 2 follows from Lemma 5 ,

\section{REFERENCES}

[1] D. Benko, Approximation by weighted polynomials, J. Approx. Theory 120 (2003), no. 1, 153-182. MR.1954937 (2004d:41050)

[2] D. Benko, The support of the equilibrium measure, Acta Sci. Math. (Szeged) 70 (2004), no. 1-2, 35-55. MR2071963 (2005c:31002)

[3] D. Benko, S.B. Damelin, P.D. Dragnev, On the support of the equilibrium measure for arcs of the unit circle and for real intervals, Electron. Trans. Numer. Anal. 25 (2006), 27-40. MR2280361 (2008b:31003)

[4] A. Kroó, J. Szabados, On density of homogeneous polynomials on convex and star-like surfaces in $\mathbb{R}^{d}$, East J. Approx 11 (2005), 381-404. MR2189221|(2006i:41027)

[5] A.B.J. Kuijlaars, A note on weighted polynomial approximation with varying weights, J. Approx. Theory 87 (1) (1996), 112-115. MR1410614 (97k:41010) 
[6] N.I. Muskhelishvili, Singular Integral Equations. Dover, New York, 1992. MR.1215485 (94a:45001)

[7] E.B. Saff, Incomplete and orthogonal polynomials. In C.K. Chui, L.L Schumaker, and J.D. Ward, editors, Approximation Theory IV, 219-255, Academic Press, New York, 1983. MR.754347 (86b:41029)

[8] E.B. Saff, V. Totik, Logarithmic Potentials with External Fields, Springer-Verlag, Berlin, 1997. MR.1485778 (99h:31001)

[9] P. Simeonov, A minimal weighted energy problem for a class of admissible weights, Houston J. of Math. 31 (2005), no. 4, 1245-1260. MR2175434(2006i:31002)

[10] A. F. Timan, Theory of Functions of a Real Variable, (Moscow, 1960) (in Russian).

[11] V. Totik, Weighted polynomial approximation for convex external fields, Constr. Approx. 16 (2) (2000) 261-281. MR.1735243 (2000j:41010)

[12] V. Totik, Weighted approximation with varying weight. Lecture Notes in Mathematics, 1569, Springer-Verlag, Berlin, 1994. MR,1290789 (96f:41002)

[13] P. Varjú, Approximation by homogeneous polynomials, Constr. Approx. 26 (2007), no. 3, 317-337. MR2335686

Department of Mathematics, Western Kentucky University, Bowling Green, KenTUCKY 42101

Current address: Department of Mathematics and Statistics, ILB 325, University of South Alabama, Mobile, Alabama 36688

E-mail address: dbenko@jaguar1.usouthal.edu

Alfréd Rényi Institute of Mathematics, Hungarian Academy of Sciences, H-1053 Budapest, ReÁltanoda u. 13-15, Hungary

E-mail address: kroo@renyi.hu 